\title{
Holographic entanglement entropy in Lovelock gravities
}

\author{
Jan de Boer, ${ }^{a}$ Manuela Kulaxizi ${ }^{b}$ and Andrei Parnachev ${ }^{c}$ \\ ${ }^{a}$ Institute for Theoretical Physics, University of Amsterdam, \\ Science Park 904, Postbus 94485, 1090 GL Amsterdam, The Netherlands \\ ${ }^{b}$ Theoretical Physics, Department of Physics and Astronomy, \\ Uppsala University, Box 516, SE-751 20 Uppsala, Sweden \\ ${ }^{c}$ Institute of Cosmos Sciences and E.C.M., Facultat de Fisica, \\ Universitat de Barcelona, Av. Diagonal 647, 08028 Barcelona, Spain \\ E-mail: J.deBoer@uva.nl, manuela.kulaxizi@fysast.uu.se, \\ parnachev@gmail.com
}

ABSTRACT: We study entanglement entropies of simply connected surfaces in field theories dual to Lovelock gravities. We consider Gauss-Bonnet and cubic Lovelock gravities in detail. In the conformal case the logarithmic terms in the entanglement entropy are governed by the conformal anomalies of the CFT; we verify that the holographic calculations are consistent with this property. We also compute the holographic entanglement entropy of a slab in the Gauss-Bonnet examples dual to relativistic and non-relativistic CFTs and discuss its properties. Finally, we discuss features of the entanglement entropy in the backgrounds dual to renormalization group flows between fixed points and comment on the implications for a possible c-theorem in four spacetime dimensions.

KEYwords: Gauge-gravity correspondence, Anomalies in Field and String Theories ARXIV EPRINT: 1101.5781 


\section{Contents}

1 Introduction and summary $\quad 1$

2 Entanglement entropy and conformal field theories in four dimensions. 2

3 Gauss-Bonnet gravity 4

4 Holographic entanglement entropy proposal $\quad 6$

4.1 The entanglement entropy of a ball 8

4.2 The entanglement entropy of a cylinder 9

$\begin{array}{ll}4.3 \text { The entanglement entropy of a slab } & 10\end{array}$

5 Generalization to Lovelock $\quad 12$

6 Entanglement entropy in Lifshitz backgrounds $\quad 13$

$\begin{array}{ll}\text { 6.1 Entanglement entropy of an infinite belt } & 14\end{array}$

6.2 Entanglement entropy of an infinitely long cylinder 16

7 Entanglement entropy and domain wall geometries $\quad 17$

$\begin{array}{ll}\text { 7.1 The entanglement entropy of a ball along the RG flow } & 18\end{array}$

$\begin{array}{ll}\text { 7.2 The entanglement entropy of a cylinder along the RG flow } & 18\end{array}$

$\begin{array}{lll}7.3 \beta_{1} \text { and the weak energy condition } & 19\end{array}$

7.4 Entanglement entropy of the ball and the c-theorem in Einstein-Hilbert gravity 20

\section{Introduction and summary}

Entanglement entropy is an interesting non-local observable which carries important information about field theory. Refs. [1,2] proposed a way of computing entanglement entropy in the strongly coupled conformal field theories dual to gravitational theories whose gravity sector is described by the Einstein-Hilbert lagrangian with the negative cosmological constant. The set of CFTs that admit duals of this type is strongly restricted. In particular in four spacetime dimensions, all such CFTs necessarily have their $a$ and $c$ central charges equal to each other.

It is known that some interesting phenomena in CFTs and, more generally, in quantum field theories, are associated with the regime where $a \neq c$. For example, there are unitarity constraints in CFTs [3] which restrict the ratio of $a / c$ to lie within certain bounds. Another interesting and important question is whether one can formulate and prove the analog of Zamolodchikov's c-theorem [4] in three and more spacetime dimensions. It has been suggested that the value of the c-function in four dimensions is equal to $a$ at fixed 
points [5]; recent work in field theory includes [6-8]. The conjecture is known by the name "a-theorem".

Holographic theories with higher derivative terms provide a natural arena for investigating these phenomena. Recently Myers and Sinha $[9,10]$ have shown that one can formulate an a-theorem in certain higher derivative theories of gravity, but it is not completely clear what the field theoretic counterpart of the corresponding a-function is. Their results have been generalized to Lovelock theories in [11]. (Work which uses higher derivative gravitational theories to study unitarity constraints in CFTs includes [12-25].) Interestingly, entanglement entropy provides an independent way of computing the $a$ and $c$ central charges in the CFTs. In particular, it has been noticed in [26] that depending on the shape of the surface which defines entanglement entropy, the logarithmic terms contain a linear combination of the $a$ and $c$ central charges. This provides an additional motivation to investigate the holographic entanglement entropy (EE) in the theories with higher derivative gravitational terms. In this paper we consider Lovelock gravities, paying special attention to the Gauss-Bonnet and cubic Lovelock cases. We make use of the prescription of [27] and generalize it to the Lovelock case to compute the logarithmic terms in the holographic entanglement entropy for a few simple geometries such as a ball, a cylinder and a slab.

The rest of the paper is organized as follows. In the next section we review the results of [26] which imply that EE of a ball contains a logarithmic term proportional to the $a$ central charge, while the $\mathrm{EE}$ of a cylinder in the similar manner encodes the $c$ central charge. In section 3 we give a brief review of Lovelock theories of gravity. Section 4 contains the description of holographic entropy proposal of $[1,2]$ together with the generalization to the Gauss-Bonnet case [27]. There we holographically compute the logarithmic terms in the EE of a ball and a cylinder in CFTs dual to Gauss-Bonnet gravity in $A d S_{5}$ and confirm that they are proportional to the $a$ and $c$ central charges respectively. We also compute the entanglement entropy of a slab as a function of Gauss-Bonnet parameter. In section 5 we make an educated guess for the holographic formula valid in all Lovelock theories. We show that the coefficient of the logarithmic term in the EE of a cylinder in six-dimensional CFT dual to cubic Lovelock gravity in $A d S_{7}$ is proportional to the linear combination of the B-type anomaly coefficients. In section 6 we consider the solution in the bulk which is holographically dual to the non-relativistic field theory with Lifshitz symmetries. We compute the entanglement entropy of a slab and a cylinder and compare with earlier results. Section 7 is devoted to studying entanglement entropy in the bulk geometries dual to renormalization group flows between conformal fixed points, motivated by the search for a c-theorem in four spacetime dimensions.

Note added: as we were working on this project, we became aware of the forthcoming paper [28] which partially overlaps with our results (see also [29]).

\section{Entanglement entropy and conformal field theories in four dimensions.}

The entropy of entanglement (EE) in a $d$-dimensional quantum field theory on $\mathbb{R}_{d-1} \times \mathbb{R}$ is defined as the von Neumman entropy of the reduced density matrix associated with a 
subspace $V$ of the total space $\mathbb{R}_{d-1}$ where the field theory lives

$$
S(V)=-\operatorname{tr}_{V} \rho_{V} \ln \rho_{V}
$$

The EE can be ultraviolate (UV) divergent in the continuum limit and a cutoff $\epsilon$ needs to be introduced. The leading divergent term is usually proportional to the area of the boundary of $V,(\partial V)$

$$
S(V) \sim \gamma \frac{\operatorname{Area}(\partial V)}{\epsilon^{d-2}}+\mathcal{O}\left(\frac{1}{\epsilon^{d-3}}\right)
$$

where the proportionality coefficient $\gamma$ depends on the regularization procedure. This result, known as the "area law" for EE, was first found numerically [30, 31] and later derived analytically [32-37]. Note however that the area law is violated in the presence of a finite Fermi surface [38-41].

For $d$-dimensional conformal field theories (CFTs) the structure of the divergent terms usually takes the following form [42]

$$
S(V)=\frac{g_{d-2}[\partial V]}{\epsilon^{d-2}}+\cdots+\frac{g_{1}[\partial V]}{\epsilon}+g_{0}[\partial V] \ln \epsilon+s(V) .
$$

Here, $s(V)$ is the finite part of the entropy and $g_{i}[\partial V]$ are local, homogeneous of degree $i$, functions of the characteristic length scale of the boundary $(\partial V)$. Eq. (2.3) is based both on the local nature of the ultraviolate divergences and on the fact that regions with common boundary share the same entropy. In general, the terms $g_{d-2}[\partial V], \cdots, g_{1}[\partial V]$ are not physical and depend on the regularization procedure. On the other hand, the coefficient of the logarithmic term is physical and universal in nature, not affected by cutoff redefinitions.

Here our primary focus will be on conformal field theories in $d=4$ dimensions where the universal coefficient of EE was recently obtained [26]. In particular, using the replica trick and conformal invariance of a four dimensional CFT on a curved manifold, [26] proposed that the coefficient of the logarithmically divergent term in the entanglement entropy of a smooth and connected region $V$ is given by

$$
g_{0}[\partial V]=\frac{c}{720 \pi} g_{0 c}[\partial V]-\frac{a}{720 \pi} g_{0 a}[\partial V]
$$

where $(c, a)$ are the central charges of the four dimensional CFT and $g_{0 c}, g_{0 a}$ are defined as follows

$$
\begin{aligned}
& g_{0 c}[\partial V]=\int_{\partial V} R_{\mu \nu \sigma \tau}\left(n_{i}^{\mu} n_{i}^{\sigma}\right)\left(n_{j}^{\nu} n_{j}^{\tau}\right)-R_{\mu \nu} n_{i}^{\mu} n_{i}^{\nu}+\frac{1}{3} R+\mu\left[\frac{1}{2} k^{i} k^{i}-\left(k_{\mu \nu}^{i}\right)^{2}\right] \\
& g_{0 a}[\partial V]=\int_{\partial V} R_{(\partial V)}=\int_{\partial A} R_{\mu \nu \sigma \tau}\left(n_{i}^{\mu} n_{i}^{\sigma}\right)\left(n_{j}^{\nu} n_{j}^{\tau}\right)-2 R_{\mu \nu} n_{i}^{\mu} n_{i}^{\nu}+R+\left[k^{i} k^{i}-\left(k_{\mu \nu}^{i}\right)^{2}\right] .
\end{aligned}
$$

Notice that $g_{0 a}[\partial V]$ is simply the Euler character of the boundary manifold $(\partial V)$ which we have also expressed (with the help of the Gauss-Codazzi identity) in terms of the ambient spacetime Riemann $R_{\mu \nu \sigma \tau}$ and Ricci $R_{\mu \nu}$ curvatures. $g_{0 c}[\partial V]$ on the other hand, to the best of our knowledge does not have a clear geometric meaning.

Let us clarify the notation used in (2.5). Suppose the two dimensional boundary $(\partial V)$ is parameterized by a set of coordinates $x^{a}$ with $a=1,2$ whereas the spacetime metric $g_{\mu \nu}$ 
where the CFT lives is spanned by coordinates $X^{\mu}$ with $\mu=0,1,2,3$. Then, $n_{i}^{\mu}$ in (2.5) denote two $(i=1,2)$ vectors normal to the surface $(\partial V)$ satisfying:

$$
\begin{aligned}
n_{i}^{\mu} n_{j}^{\nu} g_{\mu \nu} & =\delta_{i j} \\
g_{\mu \nu} \frac{\partial X^{\mu}}{\partial x^{a}} n_{i}^{\nu} & =0 .
\end{aligned}
$$

$k_{\mu \nu}^{i}$ represents the extrinsic curvature tensor of $(\partial V)$ associated to the normal $n_{i}$ and is given by

$$
k_{\mu \nu}^{i}=-\gamma_{\mu}^{\rho} \gamma_{\nu}^{\sigma} \nabla_{\rho} n_{\sigma}^{i},
$$

where $\gamma_{\mu \nu}$ represents the induced metric equal to $\gamma_{\mu \nu}=g_{\mu \nu}-n_{\mu}^{i} n_{\nu}^{i}$. Note that $\mu, \nu=$ $0,1,2,3$ are spacetime indices raised and lowered with the metric $g_{\mu \nu}$. Finally, $k^{i}$ is the trace of the extrinsic curvature tensor $k^{i}=k_{\mu \nu}^{i} g^{\mu \nu}$.

The coefficient $\mu$ in (2.5) cannot be determined by conformal invariance. In [26] it is fixed by requiring agreement between (2.4) and the holographic calculation. Using this result [26] concluded that the coefficient of the logarithmic term in the entanglement entropy of a ball $B$ and a cylinder $C$ in any four dimensional conformal field theory takes the form

$$
\begin{aligned}
& S(B)=\cdots+\frac{a}{90} \ln \epsilon+s(B) \\
& S(C)=\cdots+\frac{c}{720} \frac{l}{R} \ln \epsilon+s(C)
\end{aligned}
$$

where $B, C$ denote a ball of radius $R$ and an infinite cylinder of radius $R$ and length $l$ respectively. ${ }^{1}$ This interesting result provides an additional characterization of the anomaly coefficients $(c, a)$ through entanglement entropy.

Independent evidence in support of the work of [26] was first given in [43]. The authors of [43] used Srednicki's regularization method and numerically computed the coefficient of the logarithmic term in the entanglement entropy of a ball for a free bosonic CFT in $d=4$ dimensions. Their result was in complete agreement with [26]. Recently, [44] analytically computed the entanglement entropy for the region of a ball in a massless scalar field theory in arbitrary dimensions, further verifying Solodukhin's formula for this case. General results for the entanglement entropy for a spherical region were established later on in [45-48].

It is interesting to use eqs $(2.4),(2.5)$ to compute the coefficients of the logarithmic term of the EE for spatial regions of different geometrical shape such as, ellipsoids, toroids e.t.c. It is important to stress here that the results of [26] are restricted to regions $V$ of smooth geometrical shape. Otherwise, contributions from the non-smooth boundary are likely to modify the coefficients of the logarithmically divergent terms in the entropy [42].

\section{Gauss-Bonnet gravity}

Among all theories of gravity which contain higher derivative terms of the Riemann tensor in the their action there exists a special class of theories usually referred to, as Lovelock

\footnotetext{
${ }^{1}$ Note that for a region $V$ with zero extrinsic curvature, e.g. a slab, the logarithmic term vanishes.
} 
gravity. This class of gravitational theories stands out both for its simplicity and the several properties it shares with Einstein-Hilbert gravity. In particular, it is the most general theory of gravity whose equations of motion involve only second order derivatives of the metric. It is ghost free when expanded around a Minkowski spacetime background, while recently, the Palatini and metric formulations of Lovelock gravity have been shown to be equivalent [49].

The action for Lovelock gravity in $d+1$-dimensions is

$$
S=\frac{1}{16 \pi G_{N}^{d+1}} \int d^{d+1} x \sqrt{-g} \sum_{p=0}^{\left[\frac{d}{2}\right]}(-)^{p} \frac{(d-2 p) !}{(d-2) !} \lambda_{p} \mathcal{L}_{p},
$$

where $G_{N}^{d+1}$ is the $d+1$-dimensional Newton's constant, $\left[\frac{d}{2}\right]$ denotes the integral part of $\frac{d}{2}$, $\lambda_{p}$ is the $p$-th order Lovelock coefficient ${ }^{2}$ and $\mathcal{L}_{p}$ is the Euler density of a $2 p$-dimensional manifold. In $d+1$ dimensions all $\mathcal{L}_{p}$ terms with $p \geq\left[\frac{d}{2}\right]$ are either total derivatives or vanish identically.

In this work we will primarily focus on five dimensional gravitational theories and limit ourselves to the Gauss-Bonnet action. This is the simplest example of a Lovelock action, with only the 4-dimensional Euler density included

$$
S=\frac{1}{16 \pi G_{N}^{(5)}} \int d^{5} x \sqrt{-g}\left(R+\frac{12}{L^{2}}+\frac{\lambda L^{2}}{2} \mathcal{L}_{(2)}\right) .
$$

Note that in eq. (3.2) we introduced a cosmological constant term $\Lambda=-\frac{12}{L^{2}}$ and denoted the dimensionless Gauss-Bonnet parameter by $\lambda$ instead of $\lambda_{2}$ since the other Lovelock terms vanish in this case. In what follows we will retain this notation except for section 5 , where we discuss generic Lovelock theories. The Gauss-Bonnet term $\mathcal{L}_{(2)}$ in $(3.2)$ is

$$
\mathcal{L}_{(2)}=R_{M N P Q} R^{M N P Q}-4 R_{M N} R^{M N}+R^{2}
$$

Equations of motion derived from (3.2) are expressed in the following way

$$
-\frac{1}{2} g_{M N} \mathcal{L}+R_{M N}+\lambda L^{2} \mathcal{H}_{M N}^{(2)}=0
$$

with $\mathcal{H}_{M N}^{(2)}$ defined as

$$
\mathcal{H}_{M N}^{(2)}=R_{M L P Q} R_{N}{ }^{L P Q}-2 R_{M P} R_{N}{ }^{P}-2 R_{M P N Q} R^{P Q}+R R_{M N}
$$

Eq. (3.4) admits AdS solutions of the form [50, 51]

$$
d s^{2}=\frac{L_{\mathrm{AdS}}^{2} d r^{2}}{r^{2}}+\frac{r^{2}}{L_{\mathrm{AdS}}^{2}}\left(-d t^{2}+\sum_{i=1}^{d-1} d x_{i} d x^{i}\right)
$$

where the curvature scale of the AdS space is related to the cosmological constant via ${ }^{3}$

$$
L_{\mathrm{AdS}}=\frac{L}{\sqrt{\alpha}} \quad \alpha=\frac{2}{1+\sqrt{1-4 \lambda}} .
$$

\footnotetext{
${ }^{2}$ Note that $\lambda_{p}$ are denoted as $\hat{\lambda}_{p}$ in $[19]$.

${ }^{3}$ To be specific, Gauss-Bonnet gravity admits another AdS solution with $\alpha=\frac{2}{1-\sqrt{1-4 \lambda}}$ but this solution is unstable and contains ghosts [50].
} 
Gauss-Bonnet gravity has been extensively studied in the context of the AdS/CFT correspondence. The basic aspects of the holographic dictionary established in the case of Einstein-Hilbert gravity remain the same, since the equations of motion retain their second order form. Moreover, the additional parameter $\lambda$ allows for a holographic CFT dual with unequal central charges $(c, a)$. It thus provides an opportunity to investigate several new aspects of the correspondence (recall that all AdS backgrounds satisfying the Einstein-Hilbert equations of motion yield $a=c$ ).

There are two ways to relate the gravitational parameters, the Gauss-Bonnet coupling $\lambda$, Newton's five dimensional coupling constant $G_{N}^{(5)}$ and the cosmological constant $L$, to the CFT parameters $(c, a)$. One is via a holographic calculation of the three point function of the stress energy tensor and the other through the holographic computation of the Weyl anomaly $[52,53]$. Both calculations yield the same result, which is a good consistency check. The holographic calculation of the Weyl anomaly in Gauss-Bonnet gravity was performed in [54]. Here we simply quote the results

$$
\begin{aligned}
& c=45 \pi \frac{L_{\mathrm{AdS}}^{3}}{G_{N}^{(5)}} \sqrt{1-4 \lambda} \\
& a=45 \pi \frac{L_{\mathrm{AdS}}^{3}}{G_{N}^{(5)}}[-2+3 \sqrt{1-4 \lambda}],
\end{aligned}
$$

where $L_{\mathrm{AdS}}$ is given from (3.7). In our conventions the CFT central charges $(c, a)$ are defined through the Weyl anomaly in the following way

$$
T_{\mu}^{\mu}=\frac{1}{64 \pi^{2}} \frac{1}{90}\left(c I-a \mathcal{L}_{2}\right)
$$

It will be helpful for the calculations in the next section to have the ratio $\frac{L_{\mathrm{AdS}}^{3}}{G_{N}^{(5)}}$ and the Gauss-Bonnet coefficient, $\lambda$, expressed as functions of the central charges $(c, a)$

$$
\begin{aligned}
\frac{L_{\mathrm{AdS}}^{3}}{G_{N}^{(5)}} & =\frac{1}{90 \pi}(3 c-a), \\
\lambda & =\frac{(a-5 c)(a-c)}{4(a-3 c)^{2}} \\
\sqrt{1-4 \lambda} & =\frac{2 c}{3 c-a} .
\end{aligned}
$$

Finally, we should note that the correspondence between the positivity of the energy flux in a CFT [3] and causality of the boundary theory in Gauss-Bonnet gravity discussed in $[12-15]$, restricts the values of the Gauss-Bonnet parameter $\lambda$ to lie within the region $-\frac{7}{36} \leq \lambda \leq \frac{9}{100}$. Similar results were obtained for generic Lovelock theories of gravity in arbitrary dimensions [16-21, 25].

\section{Holographic entanglement entropy proposal}

In the context of holography, entanglement entropy received a lot of attention after the work of Ryu and Takayanagi where a concrete proposal for evaluating the entanglement entropy 
was set forth $[1,2]$. In particular, the authors of $[1,2]$ conjectured that the entanglement entropy of a spatial ${ }^{4}$ region $V$ in a $d$-dimensional CFT admitting a dual description in terms of Einstein-Hilbert gravity is given by

$$
S(V)=\frac{1}{4 G_{N}^{(d+1)}} \int_{\Sigma} \sqrt{\sigma}
$$

where $\Sigma$ is defined as the minimal area surface which asymptotes to the boundary of the spatial region $V,(\partial V)$. For more details the reader is encouraged to consult [56].

This proposal has by now passed several tests. When for instance, the spatial region $V$ extends to the whole of space, entanglement entropy should coincide with statistical entropy. Indeed, at finite temperature eq. (4.1) naturally reduces to the Bekenstein-Hawking entropy formula whereas for vanishing temperature, the dual gravitational description contains no horizon and the entropy vanishes as it should. Other properties of the entanglement entropy like strong subadditivity or the fact that $V$ and its complement $V_{c}$ have the same entropy, are also satisfied by the holographic EE formula [57]. Moreover, precise agreement between the holographic computation and the field theoretic one has been shown in the cases where explicit results are available (mostly for two dimensional CFTs) [58-60].

It is interesting to generalize (4.1) to include higher derivative gravitational theories. The most natural idea is to replace eq. (4.1) with Wald's entropy formula. In fact, for GaussBonnet gravity, such a proposal already exists in the literature $[27] .{ }^{5}$ To be specific, the author of [27] suggested that the entanglement entropy of a connected region $V$ of the dual CFT, can be computed in the case of Gauss-Bonnet gravity through the following formula

$$
S(V)=\frac{1}{4 G_{N}^{(5)}} \int_{\Sigma} \sqrt{\sigma}\left(1+\lambda L^{2} R_{\Sigma}\right)
$$

Here the integral is evaluated on $\Sigma$, the three dimensional surface which at the boundary of the holographic space reduces to the two dimensional boundary $(\partial V)$ of the region whose entropy we want to compute and which is determined by minimizing (4.2). $\sigma$ in the same expression, corresponds to the determinant of the induced metric on $\Sigma$ whereas $R_{\Sigma}$ is the induced scalar curvature of $\Sigma .^{6}$

To summarize the main reasoning of [27] recall that to compute the entanglement entropy on the CFT side, one starts by evaluating the partition function on a $d$-dimensional $n$-sheeted space - formed by gluing the $n$-copies of $\mathbb{R}_{d}$ along the boundary $(\partial V)$. This procedure produces a space $\mathcal{R}_{n}$ with conical singularities on the surface $(\partial V)$. To evaluate the partition function on $\mathcal{R}_{n}$ holographically, it is necessary to identify the dual $d+1$ dimensional geometry, $\mathcal{S}_{n}$. The latter should be a solution of the gravitational action with non-zero cosmological constant, which asymptotes to $\mathcal{R}_{n}$ at the boundary. Finding such a

\footnotetext{
${ }^{4}$ Generalization to the covariant case is discussed in [55].

${ }^{5}$ For related work on holographic entanglement entropy and higher curvature corrections see also [61].

${ }^{6}$ To make the variational problem well-defined a boundary term should in principle be added in (4.2). This term does not affect the solution of the embedding surface but it changes the value of the action evaluated on the solution and thus of the entanglement entropy. It turns out however that the boundary term only modifies the leading UV-divergent term in the entanglement entropy.
} 
solution is a difficult task. Instead, [27] assumed that $\mathcal{S}_{n}$ is given by a $n$-sheeted $\operatorname{AdS}_{d+1}$ formed by gluing together $n$-copies of $\mathrm{AdS}_{d+1}$ along a surface of codimension two. Then, the problem essentially reduced to that of evaluating the gravitational action functional on a space with conical singularities. A method for performing this calculation (at least in some cases) has been developed in [62] (see also [63-70]). With the use of the above method for Einstein-Hilbert gravity, [27] arrived at the holographic entanglement entropy formula of Ryu and Takayanagi. Considering Gauss-Bonnet gravity instead, leads to the modified expression (4.2).

Recently [71] questioned some of the assumptions that were used in [27] to derive (4.2). Still, (4.2) remains a reasonable generalization of (4.1) to Gauss-Bonnet gravity. First, the proposal agrees with Wald's entropy formula for AdS-Schwartzchild black holes in Gauss-Bonnet gravity [72-75]. Therefore, whenever the spatial region $V$ coincides with the total space, the entanglement entropy is guaranteed to be equal to the thermal entropy. Moreover, the strong subadditivity property of EE is satisfied [57]. Finally, the difference between (4.2) and (4.1) is the integral of a topological quantity i.e., the euler density in two dimensions, just like the difference between the Einstein-Hilbert and Gauss-Bonnet lagrangian is the Euler density term of four dimensions.

In the following we will use the proposal of [27] to compute the entanglement entropy for a region bounded by ball, a cylinder and a slab. Comparison with (2.8) will provide yet another check of (4.2).

\subsection{The entanglement entropy of a ball}

To compute the entanglement entropy of a ball of radius $R$, it is useful to parameterize the AdS space in the following form

$$
d s_{\mathrm{AdS}}^{2}=L_{\mathrm{AdS}}^{2}\left[\frac{d \rho^{2}}{4 \rho^{2}}+\frac{1}{\rho}\left(-d t^{2}+d r^{2}+r^{2} d \Omega_{2}^{2}\right)\right] .
$$

The first step is to identify a three dimensional surface in the bulk of AdS which reduces to a sphere of radius $R$ at the boundary. Taking into account the symmetries of the problem we see that the surface in question is determined by a single function $r(\rho)$. With this ansatz the induced metric of the surface can be written as follows

$$
d s_{E E}^{2}=L_{\text {AdS }}^{2}\left\{\frac{1}{4 \rho^{2}}\left[1+4 \rho\left(\frac{\partial r}{\partial \rho}\right)^{2}\right] d \rho^{2}+\frac{r^{2}}{\rho} d \Omega_{2}^{2}\right\} .
$$

Using (4.4) to compute the induced curvature $R_{\Sigma}$ and substituting into (4.2) yields

$$
S(B)=\frac{L_{\mathrm{AdS}}^{3} \Omega_{2}}{4 G_{N}^{(5)}} \int d \rho \frac{r^{2} \sqrt{1+4 \rho\left(r^{\prime}\right)^{2}}}{2 \rho^{2}}[1+\lambda \alpha \widehat{R}],
$$

where $\alpha$ is given in (3.7) and $\widehat{R}$, the induced scalar curvature in units of the AdS radius, is

$$
\widehat{R}=\frac{2\left[\rho+4 \rho^{2}\left(r^{\prime}\right)^{2}+4 \rho r\left(r^{\prime}+8 \rho\left(r^{\prime}\right)^{3}-2 \rho r^{\prime \prime}\right)-r^{2}\left(3+20 \rho\left(r^{\prime}\right)^{2}+16 \rho^{2} r^{\prime} r^{\prime \prime}\right)\right]}{r^{2}\left[1+4 \rho\left(r^{\prime}\right)^{2}\right]^{2}} .
$$


Eq. (4.5) gives the equations of motion which determine $r(\rho)$. To specify the coefficient of the logarithmic term it suffices to solve for $r(\rho)$ to the next to leading order in the neighborhood of the boundary $\rho=0$. We find that

$$
r(\rho)=R-\frac{\rho}{2 R}+\cdots .
$$

The solution is identical in this order to the case $\lambda=0$. Substituting (4.7) into (4.5) yields

$$
S(B)=\frac{L_{\mathrm{AdS}}^{3} \Omega_{2}}{4 G_{N}^{(5)}} \int_{\epsilon^{2}} d \rho\left[\frac{1-6 \lambda \alpha}{2 \rho^{2}} R^{2}-\frac{1-6 \lambda \alpha}{4 \rho}+\mathcal{O}\left(\rho^{0}\right)\right] .
$$

Using (3.10) and the definition of $\alpha$ from (3.7) we arrive at

$$
S(B)=\frac{a}{90} \frac{R^{2}}{\epsilon^{2}}+\frac{a}{90} \ln \epsilon+\cdots,
$$

which is in complete agreement with (2.8).

\subsection{The entanglement entropy of a cylinder}

Consider a three dimensional surface in AdS which reduces to a two dimensional cylindrical surface of radius $R$ and length $l$ on the boundary of the AdS space. It is then natural to parameterize the AdS metric as follows

$$
d s_{\mathrm{AdS}}^{2}=L_{\mathrm{AdS}}^{2}\left[\frac{d \rho^{2}}{4 \rho^{2}}+\frac{1}{\rho}\left(-d t^{2}+d z^{2}+d r^{2}+r^{2} d \phi^{2}\right)\right]
$$

The symmetries of the problem lead us to consider a surface described by a single function $r(\rho)$. The induced metric is then

$$
d s_{E E}^{2}=L_{\text {AdS }}^{2}\left\{\frac{1}{4 \rho^{2}}\left[1+4 \rho\left(\frac{\partial r}{\partial \rho}\right)^{2}\right] d \rho^{2}+\frac{1}{\rho} d z^{2}+r^{2} \phi^{2}\right\} .
$$

Plugging this ansatz into (4.2) yields

$$
S(C)=\frac{L_{\mathrm{AdS}}^{3}}{4 G_{N}^{(5)}} 2 \pi l \int d \rho \frac{r \sqrt{1+4 \rho\left(r^{\prime}\right)^{2}}}{2 \rho^{2}}[1+\alpha \lambda \widehat{R}],
$$

where now $\widehat{R}$ is the induced curvature of the surface in units of the AdS radius is

$$
\widehat{R}=\frac{2\left[2 \rho\left(r^{\prime}+8 \rho\left(r^{\prime}\right)^{3}-2 \rho r^{\prime \prime}\right)-r\left(3+20 \rho\left(r^{\prime}\right)^{2}+16 \rho^{2} r^{\prime} r^{\prime \prime}\right)\right]}{r\left[1+4 \rho\left(r^{\prime}\right)^{2}\right]^{2}} .
$$

The equations of motions in the vicinity of $\rho=0$ are satisfied by ${ }^{7}$

$$
r(\rho)=R-\frac{\rho}{4 R}+\cdots .
$$

\footnotetext{
${ }^{7}$ Notice that the solution is again identical in this order to the case $\lambda=0$.
} 
Substituting (4.14) into (4.12) yields

$$
S(C)=\frac{L_{\mathrm{AdS}}^{3}}{4 G_{N}^{(5)}} 2 \pi l \int_{\epsilon^{2}} d \rho\left[\frac{(1-6 \alpha \lambda) R}{2 \rho^{2}}-\frac{1-2 \alpha \lambda}{16 R \rho}+\mathcal{O}\left(\rho^{0}\right)\right] .
$$

With the help of (3.10) and (4.13) we arrive at

$$
S(C)=\frac{a}{90} \frac{2 \pi R l}{4 \pi \epsilon^{2}}+\frac{c}{720} \frac{l}{R} \ln \epsilon+\cdots .
$$

which agrees with $(2.8)$.

\subsection{The entanglement entropy of a slab}

The slab geometry corresponds to the region of space bounded by $-\frac{y}{2} \leq x^{1} \leq \frac{y}{2}$ and infinitely extended along the $x^{2}, x^{3}$ directions. This is the simplest configuration to consider because of the large amount of symmetry. Here, it is convenient to write the AdS metric as

$$
d s^{2}=\frac{L_{\mathrm{AdS}}^{2}}{r^{2}}\left(\eta_{i j} d x^{i} d x^{j}+d r^{2}\right)
$$

with $\eta_{i j}$ the four dimensional Minkowski metric. The three dimensional induced surface can be parametrized by a single function $x_{1}(r)$ as follows

$$
d s_{E E}^{2}=\frac{L_{\mathrm{AdS}}^{2}}{r^{2}}\left[\left(1+x_{1}^{\prime}(r)^{2}\right) d r^{2}+d x_{2}^{2}+d x_{3}^{2}\right]
$$

The induced curvature of the surface (in units of the AdS radius) is non-vanishing and equal to

$$
\widehat{R}=-2 \frac{3+3 x_{1}^{\prime}(r)^{2}+2 r x_{1}^{\prime}(r) x_{1}^{\prime \prime}(r)}{\left(1+x_{1}^{\prime}(r)^{2}\right)^{2}}
$$

where the primes indicate differentiation with respect to the radial coordinate. The Lagrangian of the system is independent of $x_{1}(r)$ so there is a constant of motion

$$
\frac{x_{1}^{\prime}(r)\left(1-2 \alpha \lambda+x_{1}^{\prime}(r)^{2}\right)}{r^{3}\left(1+x_{1}^{\prime}(r)^{2}\right)^{\frac{3}{2}}}=\frac{1}{r_{\star}^{3}}
$$

which allows us to solve for $x_{1}^{\prime}(r)$ exactly. Since the theory is conformal, one can rescale the coordinate by denoting $\tau=r / r_{*}$. Then eq. (6.5) can be written as

$$
\sqrt{h(\tau)} \frac{(1-2 \alpha \lambda+h(\tau))}{(1+h(\tau))^{\frac{3}{2}}}=\tau^{3}
$$

where $h(\tau)=x_{1}^{\prime}(r)^{2}$. It is easy to see that there are three solutions for $h(\tau)$ but only one of them is continuously connected with the solution of the $\lambda=0$ case. In the following we restrict our attention to this solution. ${ }^{8}$ It would be interesting to examine the other two solutions which at first glance appear to be complex valued. We leave this analysis to future work.

\footnotetext{
${ }^{8}$ We avoid writing down the solution explicitly since it is not particularly illuminating.
} 


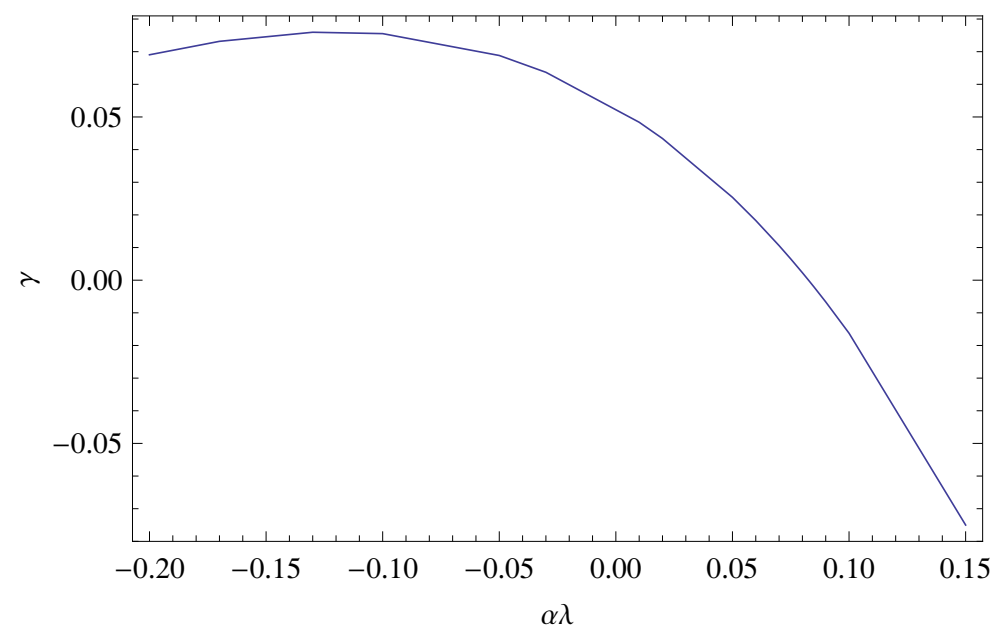

Figure 1. $\gamma(\lambda)$ [see eq. (6.8)] as a function of $\lambda \alpha$.

We proceed to relate the constant of motion $r_{\star}$ with the width $y$ of the slab

$$
\frac{y}{2}=r_{\star} I_{0}(\lambda) \quad I_{0}(\lambda)=\int_{0}^{1} d \tau \sqrt{h(\tau)} .
$$

Evaluating the action on the solution of (4.21) yields

$$
S(\lambda)=\frac{L_{\mathrm{AdS}}^{3}}{4 G_{N}^{(5)}}\left\{\frac{1-6 \alpha \lambda}{2} \frac{l^{2}}{\epsilon^{2}}+\gamma(\lambda) \frac{l^{2}}{y^{2}}\right\}
$$

where

$$
\gamma(\lambda)=4 I_{0}(\lambda)^{2} \int_{0}^{1} d \tau\left[\frac{[1+h(\tau)]^{\frac{1}{2}}}{\tau^{3}}\left(1-\frac{2 \alpha \lambda\left(3+3 h(\tau)+\tau h^{\prime}(\tau)\right)}{[1+h(\tau)]^{2}}\right)-\frac{(1-2 \alpha \lambda)}{\tau^{3}}\right]
$$

Recall from (3.10) that $\lambda$ can be expressed as a function of the ratio $\frac{a}{c}$ whereas $\frac{L_{\mathrm{AdS}}^{3}}{4 G_{N}^{(5)}}=$ $\frac{3 c-a}{4 \times 90 \pi}$. This allows us to express the final result as

$$
S(\lambda)=\frac{1}{4 \pi} \frac{a}{90} \frac{l^{2}}{\epsilon^{2}}+\gamma(a, c) \frac{l^{2}}{y^{2}},
$$

with $\gamma(a, c)=\frac{3 c-a}{4 \pi \times 90} \gamma(\lambda)$. The result of numerical integration in eq. (6.8) is shown in figure 1.

We see that the EE of a slab has the form expected from field theory considerations. The leading divergent term follows the area law. Its coefficient is proportional to the central charge $a$ just like the entanglement entropy of the ball and the infinite cylinder. However this coefficient depends on the regularization procedure and has no physical meaning. On the other hand, the coefficient of the second term in (4.25) is universal and physical. Computing $\gamma(a, c)$ numerically we see that it changes sign approximately at $\alpha \lambda \sim .08$, or $\frac{a}{c} \sim .62$. The change of sign implies that $\gamma(a, c)$ is not proportional to the coefficient $\widetilde{c}=\frac{(3 c-a)^{4}}{(5 c-a)^{3}}$ of the thermal entropy $s=\widetilde{c} T^{3}$. Recall also that $\alpha \lambda=\frac{c-a}{2(3 c-a)}$ and from Fig 1. 
note that $\gamma(\lambda)$ is not linear in $(\alpha \lambda)$. This observation shows that $\gamma(a, c)$ is not linear in $a$ and $c$. It would be interesting to explore this further. Another direction to pursue, is to consider the Gauss-Bonnet holographic dual of a confining gauge theory. Computing the EE for a slab in this background and generalizing the results of [76] may help to clarify the meaning of $\gamma(a, c)$.

\section{Generalization to Lovelock}

So far we used Fursaev's proposal to compute the leading divergent terms in the entanglement entropy of a ball, an infinite cylinder and a slab in a four dimensional CFT defined from Gauss-Bonnet gravity via gauge-gravity duality. Gauss-Bonnet gravity allows for the holographic description of CFTs with unequal central charges $(c, a)$ giving an opportunity to check the proposal of [27] against the results of [26]. According to [26], the coefficient of the logarithmic term in the entropy should be proportional to $a$ for a ball, $c$ for an infinite cylinder and vanish for a slab, which is precisely what we found by using the prescription of [27]. This result led us to generalize the proposal of [27] to any Lovelock theory of gravity

$$
S(V)=\frac{1}{4 G_{N}^{(d+1)}} \sum_{p=0}^{\left[\frac{d}{2}\right]}(-)^{p+1}(p+1) \frac{(d-2 p-2) !}{(d-2) !} \lambda_{p+1} \int_{\Sigma} \sqrt{\sigma} \mathcal{L}_{(p)} .
$$

Expression (5.1) coincides with the expression for the entropy of black holes in Lovelock gravity as established in [72, 73], which in turn agrees with Wald's entropy formula [74, 75]. As a result (5.1) satisfies by construction several properties of the entanglement entropy.

An interesting check of the proposal (5.1) is the computation of the entanglement entropy of the cylinder in the case of cubic Lovelock gravity dual to the six-dimensional CFT. In this case, we expect the log term in the EE to be proportional to the coefficients of the B-type anomaly (just as in the four-dimensional case, the coefficient was proportional to c). ${ }^{9}$ In fact, there is a non-trivial check of this statement. In Lovelock gravity, the three coefficients of the B-type anomaly, $b_{i}, i=1, \ldots, 3$ are not linearly independent. Using the results of [19] one can write them as

$$
\begin{aligned}
& b_{1}=\widetilde{c} \frac{L_{\mathrm{AdS}}^{3}}{4 \pi G_{N}^{(7)}}\left(\frac{204}{\alpha}-168+100 \lambda_{2} \alpha\right) \\
& b_{2}=\widetilde{c} \frac{L_{\mathrm{AdS}}^{3}}{4 \pi G_{N}^{(7)}}\left(\frac{75}{\alpha}-66+41 \lambda_{2} \alpha\right) \\
& b_{3}=\widetilde{c} \frac{L_{\mathrm{AdS}}^{3}}{4 \pi G_{N}^{(7)}}\left(-\frac{9}{\alpha}+6-3 \lambda_{2} \alpha\right)
\end{aligned}
$$

where we used

$$
\lambda_{3} \alpha^{2}=-\frac{1}{\alpha}+1-\lambda_{2} \alpha
$$

\footnotetext{
${ }^{9}$ For the EE of a ball, the log term is shown to be proportional to the coefficient of the A-type anomaly in [28].
} 
to eliminate terms linear in $\lambda_{3}$. The overall coefficient $\widetilde{c}$ is related to the definition of the invariants $I_{i}, i=1, \ldots, 3$ and is therefore not important. One can fix its numerical value to be $\widetilde{c}=-1 / 2304$. We can now use (5.1) to compute the $\mathrm{EE}$ of the cylinder. In particular, (4.7) becomes

$$
r(\rho)=R-\frac{3 \rho}{8 R}+\frac{45-98 \lambda_{2} \alpha-159 \lambda_{2} \alpha^{2}}{521\left(-1+2 \lambda_{1} \alpha+3 \lambda_{2} \alpha^{2}\right)} \frac{\rho^{2}}{R^{3}}+\ldots
$$

while the result for the $\mathrm{EE}$

$$
S_{\log }=-\frac{135}{4 \times 512} \frac{L_{\mathrm{AdS}}^{5}}{4 G_{N}^{(7)}} \frac{2 \pi^{2} l}{R}\left[1-\frac{94}{45} \lambda_{2} \alpha-\frac{49}{15} \lambda_{3} \alpha^{2}\right] \ln \epsilon
$$

Using (5.3) we can eliminate the term proportional to $\lambda_{3}$ from the square brackets. The resulting expression has three terms, but turns out to be proportional to the linear combination of the two central charges (5.2),

$$
1-\frac{94}{45} \lambda_{2} \alpha-\frac{49}{15} \lambda_{3} \alpha^{2}=\frac{19}{360} b_{1}-\frac{1}{10} b_{2}
$$

which is a highly nontrivial check of (5.1). Note that the third central charge, $b_{3}$, is a linear combination of $b_{1}$ and $b_{2}$ (since Lovelock gravity was shown to satisfy the supersymmetric constraint [77]). Finally, the results of this section can be helpful in extending the proposal of $[26]$ to CFTs of higher dimensionality. We hope to investigate this issue further in the future.

\section{Entanglement entropy in Lifshitz backgrounds}

Lifshitz spacetimes are geometries of the form

$$
d s^{2}=\frac{\widehat{L}^{2}}{r^{2}}\left[d r^{2}-\frac{1}{r^{2 w-2}} d t^{2}+\sum_{i=1}^{d-1} d x_{i}^{2}\right]
$$

which preserve the scaling symmetry

$$
r \rightarrow \kappa^{-1} r, \quad t \rightarrow \kappa^{w} t, \quad x^{i} \rightarrow \kappa x^{i}, .
$$

$w$ is usually referred to as the dynamical critical exponent. It is clear that any metric of the form (6.1) with $w \neq 1$ breaks Lorentz invariance.

Lifshitz spacetimes received considerable attention in the literature (together with Schrodinger geometries) because they provide a natural playground for the study of strongly coupled systems near non-relativistic critical points [78]. Recently, they have been embedded in string theory [79-82]. This typically requires non-trivial profiles for fields other than the metric to be turned on. Including higher derivative terms in the gravitational action however changes the situation. In [83] it was argued that higher curvature terms generically lead to a finite renormalization of the critical exponent $w$ and matter fields are no longer required for the theory to support Lifshitz solutions.

In the specific case of Lovelock gravity it was shown in $[84,85]$ that for special values of the Lovelock parameters, Lifshitz solutions of arbitrary critical exponent $w$ can be constructed. ${ }^{10}$ Restricting our attention to Gauss-Bonnet gravity, we find that (6.1) solves

\footnotetext{
${ }^{10}$ Asymptotically Lifshitz black hole solutions however do not exist in pure Lovelock gravity [84].
} 
the equations of motion (3.4) as long as $\lambda=\frac{1}{4}$ and $\widehat{L}^{2}=\frac{L^{2}}{2} \cdot{ }^{11}$ Note that when $\lambda=\frac{1}{4}$ symmetries preserved by the gravitational action functional are enhanced to the $\mathrm{SO}(4,2)$ group. The Lagrangian of (3.2) then coincides with the Chern-Simons Lagrangian for the AdS group and admits a natural supersymmetrization [86]. It is perhaps worth mentioning that the AdS solution of Gauss-Bonnet gravity for $\lambda=\frac{1}{4}$ is difficult to interpret in the context of the AdS/CFT correspondence since the dual conformal field theory has vanishing central charge $c$.

Here we will focus on computing the entanglement entropy holographically in $d=4$ dimensional field theories with Lifshitz scaling. When the time coordinate is the only coordinate with a different scaling, the computation of entanglement entropy is identical to the one in AdS spacetime. Therefore the results of the previous sections go through unmodified as long as we make the substitution $\lambda \rightarrow \frac{1}{4}$ and $L_{\mathrm{AdS}} \rightarrow \frac{L}{\sqrt{2}}$. In the following we will consider the more interesting case where rotational invariance is broken and instead of the time coordinate, one of the spatial coordinates e.g. $x^{1}$, scales like $x^{1} \rightarrow \kappa^{w} x^{1}$. In particular, we will compute the entanglement entropy of an infinitely extended slab and a cylinder. Generalization to higher dimensions is straightforward.

As a final remark, let us mention here that a statement relating the universal terms in the entropy with the "central charges" (physical quantities measuring degrees of freedom) of the corresponding quantum field theory, is absent in this case (see however [10] for some attempts in this direction). We will see in section 6.2 that the general structure of the divergent terms in the entanglement entropy of a cylinder is quite different from the relativistic case. In particular, there is no logarithmic term of the form which appears in (2.8). This result might allow one to suggest that either a connection between degrees of freedom in non-relativistic theories and universal terms in the entanglement entropy can not be made or it will be of quite a different form from the relativistic one. This issue clearly deserves further investigation.

\subsection{Entanglement entropy of an infinite belt}

Consider double Wick rotation of (6.1),

$$
d s^{2}=\frac{\widehat{L}^{2}}{r^{2}}\left[d r^{2}+\frac{1}{r^{2 w-2}} d x_{1}^{2}+\left(-d t^{2}+\sum_{i=2}^{d-1} d x_{i}^{2}\right)\right]
$$

Now there are two distinct orientations for the belt geometry, depending on whether its width (smallest size) extends along the direction with $w$-scaling or not. Here we concentrate on the case where the slab is infinitely extended along the direction with anisotropic scaling.

\footnotetext{
${ }^{11}$ For completeness, we remark that Gauss-Bonnet gravity (for particular values of the parameter $\lambda$ ) admits Lifshitz solutions of the most generic form where several boundary coordinates scale like $x^{i} \rightarrow \kappa^{w} x^{i}$. However, the anisotropic scaling is a relative notion and thus, in $d=4$ there are effectively only two physically distinct cases. The first case, with a single coordinate of anisotropic scaling, is discussed above. The second case, of a Lifshitz spacetime with two coordinates scaling anisotropically, also satisfies the equations of motion of Gauss-Bonnet gravity as long as $\lambda=\frac{1+w+w^{2}}{12 w}$ and $\widehat{L}^{2}=\frac{1+w+w^{2}}{6} L^{2}$. We will not address the latter case in the following which can be studied in a similar manner.
} 
So let us consider a stripe, infinitely extended along directions $x_{1}, x_{3}$ and width $y$ along $x_{2}$. It is convenient to parametrize the bulk surface by a single function $x_{2}(r)$. The induced metric and curvature of the surface are

$$
\begin{aligned}
d s_{E E}^{2} & =\frac{L^{2}}{2 r^{2}}\left[\left(1+x_{2}^{\prime}(r)^{2}\right) d r^{2}+d x_{3}^{2}+\frac{d x_{1}^{2}}{r^{2 w-2}}\right] \\
\widehat{R} & =-2 \frac{\left(1+w+w^{2}\right)+\left(1+w+w^{2}\right) x_{2}^{\prime}(r)^{2}+(1+w) r x_{2}^{\prime}(r) x_{2}^{\prime \prime}(r)}{\left(1+x_{2}^{\prime}(r)^{2}\right)^{2}}
\end{aligned}
$$

Substituting (6.4) into (4.2) results in a Lagrangian independent of $x_{2}(r)$ which leads to the following equation of motion

$$
\frac{x_{2}^{\prime}(r)\left(1-w+x_{2}^{\prime}(r)^{2}\right)}{\left(1+x_{2}^{\prime}(r)^{2}\right)^{\frac{3}{2}}}=\frac{r^{2+w}}{r_{\star}^{2+w}} .
$$

Note that $r_{\star}$ is related to the width of the slab through

$$
\frac{y}{2}=r_{\star} I_{0} \quad I_{0}=\int_{0}^{1} d \tau \sqrt{h(\tau)}
$$

where the integral $I_{0}$ is expressed in terms of the dimensionless variable $\tau=\frac{r}{r_{\star}}$. and the function $h(\tau)=\left(\frac{\partial x_{2}}{\partial r}\right)^{2}$.

Expressed in terms of $h(\tau)$ eq. (6.5) has three solutions. Only one of them is real and continuously connected to the $w=1$ case. Again we will restrict our attention to this case. Evaluating (4.2) on the particular solution of (6.5) yields the entanglement entropy

$$
S_{\text {Lif. }}(S)=\frac{2^{-\frac{3}{2}} L^{3}}{4 G_{N}^{(5)}}\left[-w \frac{l_{1} \times l_{3}}{\epsilon^{w+1}}-\gamma \frac{l_{1} \times l_{3}}{y^{w+1}}\right]
$$

where $\gamma$ is a numerical constant equal to $\gamma=4 I_{0}^{2} \times\left(\frac{-w^{2}-w}{w+1}-I\right)$. $I_{0}$ is defined in (6.6) while $I$ denotes the following integral

$$
I=\int_{0}^{1} d \tau \frac{-w^{2}-w+\left(1-w-w^{2}\right) h(\tau)+h(\tau)^{2}+\frac{1+w}{2} \tau \dot{h}(\tau)}{\tau^{3}(1+h(\tau))^{\frac{3}{2}}}-\frac{-w^{2}-w}{\tau^{3}} .
$$

Let us discuss eq. (6.7). First of all, we find that the entanglement entropy is proportional to the boundary area $l_{1} \times l_{3}$ of the belt. This is in accordance with expectations from field theory considerations [87]. We see that the logarithmically divergent term is absent just like in the relativistic case. The power of the leading divergent term depends on the critical exponent $w$ and is fixed by dimensional analysis. The coefficient of the second term in (6.7) which is physical and assumed to measure the total degrees of freedom of the system, scales in the same way the cutoff scales. It is interesting to note that the sign of the leading divergent term is negative for $w>0$ in contrast to the relativistic case. This is not in contradiction with the results of the previous sections for Gauss-Bonnet gravity, because for $w=1$, i.e., AdS space, the central charge $c$ of the dual theory vanishes and $a$ becomes negative (recall that Lifshitz solutions exist for $\lambda=\frac{1}{4}$ ). For this reason the case 
$w=1$ appears to be unphysical. Nevertheless, the leading divergent term in the entropy does not have a physical meaning and its overall sign is immaterial. We expect that a proper treatment of the boundary term would give a positive result but do not pursue this issue further here. Similar results for the entanglement entropy of a slab in a Lifshitz background were obtained in [88].

\subsection{Entanglement entropy of an infinitely long cylinder}

Another interesting case to consider is that of an infinite cylinder extended along a direction with anisotropic scaling. To make contact with sections 4.2 and 4.3 we express the Lifshitz metric in the following form

$$
d s^{2}=\frac{L^{2}}{2}\left[\frac{d \rho^{2}}{4 \rho^{2}}+\frac{1}{\rho}\left(\frac{d z^{2}}{\rho^{w-1}}-d t^{2}+d r^{2}+r^{2} d \phi^{2}\right)\right]
$$

The induced metric and scalar curvature (in units $\frac{L}{\sqrt{2}}=1$ ) of the surface are

$$
\begin{aligned}
d s_{E E}^{2}= & \left(1+4 \rho r^{\prime}(\rho)^{2}\right) \frac{d \rho^{2}}{4 \rho^{2}}+\frac{d z^{2}}{\rho^{w}}+\frac{r(\rho)^{2}}{\rho} \\
\widehat{R}= & \frac{-2\left(1+w+w^{2}\right) r(\rho)-8 \rho\left(2+2 w+w^{2}\right) r(\rho) r^{\prime}(\rho)^{2}}{r(\rho)\left(1+4 \rho r^{\prime}(\rho)^{2}\right)^{2}}+ \\
& +\frac{4 \rho r^{\prime}(\rho)\left(w+4 \rho r^{\prime}(\rho)^{2}(1+w)\right)-8 \rho^{2} r^{\prime \prime}(\rho)\left(1+2(1+w) r(\rho) r^{\prime}(\rho)\right)}{r(\rho)\left(1+4 \rho r^{\prime}(\rho)^{2}\right)^{2}}
\end{aligned}
$$

where primes denote differentiation with respect to $\rho$. Combining (4.2) and (6.10) determines the equation of motion for $r(\rho)$ which takes a rather complicated form but remains second order in derivatives. Studying the equation of motion in the vicinity of the boundary $\rho=0$ we were able to eventually determine the exact solution. We find that

$$
r(\rho)=R \sqrt{1-\frac{\rho}{R^{2}}}
$$

Note that (6.11) is independent of the critical exponent $w{ }^{12}$ Evaluating the action (4.2) for $\lambda=\frac{1}{4}$ on the solution (6.11) leads to

$$
S_{\text {Lif. }}(C)=\frac{2^{-\frac{3}{2}} L^{3}}{4 G_{N}^{(5)}} 2 \pi l_{z} \int_{\epsilon^{2}}^{\infty} d \rho \frac{w R}{2 \rho^{\frac{w+3}{2}}}\left[(w-1) \frac{\rho}{R^{2}}-(1+w)\right]
$$

where $l_{z}$ regularizes the length of the cylinder. Performing the integral in (6.12) determines the entanglement entropy to be

$$
S_{\text {Lif. }}(C)=\frac{2^{-\frac{3}{2}} L^{3}}{4 G_{N}^{(5)}} 2 \pi l_{z}\left[\frac{w R}{\rho^{\frac{w+1}{2}}}\left(1-\frac{\rho}{R^{2}}\right)\right]_{\epsilon^{2}}^{\infty} \Rightarrow w>1 \frac{2^{-\frac{3}{2}} L^{3}}{4 G_{N}^{(5)}} 2 \pi l_{z}\left[\frac{w R}{\epsilon^{w+1}}\left(-1+\frac{\epsilon^{2}}{R^{2}}\right)\right]
$$

\footnotetext{
${ }^{12}$ It is interesting to expand the solution close to the boundary $\rho=0$ as $r(\rho)=R-\frac{\rho}{2 R}-\cdots$. Note that the solution is not equal to next leading order to the embedding function for the case of a cylinder in Gauss-Bonnet gravity (section 4.2 eq. (4.14)). This shows once more that the order of limits $\lambda \rightarrow \frac{1}{4}$ and $w \rightarrow 1$ cannot be interchanged.
} 
The computation of the entanglement entropy makes sense only for $w>1 .{ }^{13}$ Recall that Lifshitz geometries are unstable for $w<1$ (see for example [89]). We see a manifestation of this fact here through the computation of entanglement entropy. As expected, the leading divergence is proportional to the area $2 \pi l_{z} R$ whereas the scaling of the cutoff is fixed by dimensional analysis. Note that the logarithmic divergence, characteristic of the entanglement entropy on the cylinder in relativistic theories, is absent here. It would be interesting to examine this point further from the field theory point of view.

\section{Entanglement entropy and domain wall geometries}

In an attempt to investigate aspects of the entanglement entropy along renormalization group trajectories, we will consider here domain wall geometries. These are asymptotically AdS spaces with a metric of the form

$$
d s^{2}=d r^{2}+e^{2 A(r)} \eta_{i j} d x^{i} d x^{j}
$$

where $\eta_{i j}$ is the Minkowski metric of the dual quantum field theory spacetime. We assume here that domain wall geometries are solutions of Gauss-Bonnet gravity with matter fields (e.g. scalars) just as they are solutions of Einstein-Hilbert gravity with matter fields. In the spirit of the AdS/CFT correspondence they correspond to renormalization group flow trajectories for the dual quantum field theories. The main difference here is that GaussBonnet gravity contains an additional dimensionless constant $\lambda$ which at the fixed points of the flow, e.g. at $r=\infty$, is expressed in terms of the ratio of the central charges $a, c$ of the dual CFT.

The central objective of this section is to explore how the universal and dimensionless coefficient of the logarithmic term in the entanglement entropy changes along the renormalization group flow. The main assumption we will rely on is that the holographic computation of entanglement entropy remains the same despite the non-trivial profile of the matter fields. In particular, we will assume that the equations of motion for the matter are second order (standard kinetic term) and that their coupling to the metric is solely through its determinant. For example, we exclude the case of a conformal coupling. To make the comparison with the results of sections 4.1 and 4.2 straightforward, we express the metric (7.1) in a different coordinate system by performing the coordinate transformation $\rho=\widetilde{L}^{2} e^{-\frac{2 r}{L}}$. This way the IR region, $r=-\infty$, is mapped to $\rho=\infty$ while the UV region, $r=\infty$, corresponds to $\rho=0$. Eq. (7.1) is expressed in the following form

$$
d s^{2}=\widetilde{L}^{2}\left[\frac{d \rho^{2}}{4 \rho^{2}}+\frac{e^{2 U(\rho)}}{\rho} \eta_{i j} d x^{i} d x^{j}\right],
$$

with $U(\rho)$ related to $A(r)$ in (7.1) through $U(r) \equiv A(r)-\frac{r}{\widetilde{L}}$, or equivalently $e^{2 A(\rho)} \equiv$ $\widetilde{L}^{2} \frac{e^{2 U(\rho)}}{\rho}$. Since the metric (7.2) is asymptotically AdS, $e^{2 U(\rho)}$ admits an expansion near the boundary $\rho=0$ of the form ${ }^{14}$

$$
e^{2 U(\rho)}=1+\beta_{1} \rho+\rho^{2}\left(\beta_{2}+\beta_{3} \ln \rho+\beta_{4} \ln ^{2} \rho\right)+\cdots .
$$

\footnotetext{
${ }^{13}$ We do not consider the case $w=1$ since its physical meaning is ambiguous as explained earlier.

${ }^{14}$ In special cases, e.g. [90], the expansion may include half integral powers of $\rho$ [91-93].
} 
The dimensionfull coefficients $\beta_{1}, \beta_{2}, \cdots$ are determined by solving the field equations order by order in $\rho$.

\subsection{The entanglement entropy of a ball along the RG flow}

To compute the entanglement entropy of a ball of radius $R$ we will take steps similar to the conformal case. Writing the domain wall metric as

$$
d s^{2}=\widetilde{L}^{2}\left[\frac{d \rho^{2}}{4 \rho^{2}}+\frac{e^{2 U(\rho)}}{\rho}\left(-d t^{2}+d r^{2}+r^{2} d \Omega_{2}^{2}\right)\right],
$$

we see that the ansatz $r(\rho)$ is still natural. The induced metric is then

$$
d s_{E E}^{2}=\widetilde{L}^{2}\left\{\frac{1}{4 \rho^{2}}\left[1+4 \rho e^{2 U(\rho)}\left(\frac{\partial r}{\partial \rho}\right)^{2}\right] d \rho^{2}+\frac{r^{2}(\rho) e^{2 U(\rho)}}{\rho} d \Omega_{2}^{2}\right\},
$$

and substituting into (4.2) we arrive at

$$
S(B)=\frac{\widetilde{L}^{3} \Omega_{2}}{4 G_{N}^{(5)}} \int d \rho \frac{r^{2}(\rho) e^{2 U(\rho)} \sqrt{1+4 e^{2 U(\rho)} \rho\left(r^{\prime}\right)^{2}}}{2 \rho^{2}}[1+\alpha \lambda \widehat{R}]
$$

where $\widehat{R}=\widehat{R}(r(\rho), U(\rho))$ is the scalar curvature of the induced three dimensional surface in units of the asymptotic AdS radius in the UV, $\widetilde{L}$. The equation of motion for $r(\rho)$ derived from (7.6) is rather complicated. In the vicinity of the boundary however it is solved by $r(\rho)=R-\frac{\rho}{2 R}+\cdots$ exactly like the case $\lambda=0, U(\rho)=0$. Knowledge of the near boundary behavior of $r(\rho)$ together with (7.3) allows us to determine the divergent terms in the entanglement entropy of a ball

$$
\begin{aligned}
S(B) & =\frac{\widetilde{L}^{3} \Omega_{2}}{4 G_{N}^{(5)}} \int_{\epsilon^{2}} d \rho\left[\frac{1-6 \alpha \lambda}{2 \rho^{2}} R^{2}-\frac{1-6 \alpha \lambda}{4 \rho}\left(1-2 \beta_{1} R^{2} \frac{1-2 \alpha \lambda}{1-6 \alpha \lambda}\right)+\cdots\right] \\
& =\frac{\widetilde{L}^{3}(1-6 \alpha \lambda)}{4 G_{N}^{(5)}} \frac{R^{2} \Omega_{2}}{2 \epsilon^{2}}+\frac{\widetilde{L}^{3} \Omega_{2}}{4 G_{N}^{(5)}} \frac{1-6 \alpha \lambda}{2}\left(1-2 \beta_{1} R^{2} \frac{1-2 \alpha \lambda}{1-6 \alpha \lambda}\right) \ln \epsilon+\cdots
\end{aligned}
$$

Here $\alpha=\frac{L^{2}}{\widetilde{L}^{2}}$. To leading order in the vicinity of $\rho=0$ it is given by (3.7).

The coefficient of the logarithmically divergent term is modified compared to the pure AdS case by the overall factor $\left(1-2 \beta_{1} R^{2} \frac{1-2 \alpha \lambda}{1-6 \alpha \lambda}\right)$ and $\alpha$ given in (3.7). Since $\frac{1-2 \alpha \lambda}{1-6 \alpha \lambda}$ is positive, the behavior of the coefficient of the logarithmic term under rescalings of the radius of the ball $R \rightarrow \Lambda R$ depends on the sign of $\beta_{1}$.

\subsection{The entanglement entropy of a cylinder along the RG flow}

The case of a cylindrical surface is dealt with in a similar manner. We write the domain wall solution as

$$
d s^{2}=\widetilde{L}^{2}\left[\frac{d \rho^{2}}{4 \rho^{2}}+\frac{e^{2 U(\rho)}}{\rho}\left(-d t^{2}+d z^{2}+d r^{2}+r^{2} d \phi^{2}\right)\right]
$$


and select an ansatz of the form $r(\rho)$ which leads to the following induced metric

$$
d s_{E E}^{2}=\widetilde{L}^{2}\left\{\frac{1}{4 \rho^{2}}\left[1+4 \rho e^{2 U(\rho)}\left(\frac{\partial r}{\partial \rho}\right)^{2}\right] d \rho^{2}+\frac{e^{2 U(\rho)}}{\rho}\left(d z^{2}+r^{2}(\rho) d \phi^{2}\right)\right\} .
$$

We subsequently substitute into (4.2) and derive an equation of motion for $r(\rho)$ which we solve near the boundary $\rho=0$. The solution $r(\rho)=R-\frac{\rho}{4 R}+\mathcal{O}\left(\rho^{2}\right)$, together with (7.3), help us obtain the divergent terms for the entanglement entropy of a cylinder

$$
\begin{aligned}
S(C) & =\frac{\widetilde{L}^{3}}{4 G_{N}^{(5)}} 2 \pi l \int_{\epsilon^{2}} d \rho\left[\frac{(1-6 \alpha \lambda) R}{2 \rho^{2}}-\frac{1-2 \alpha \lambda}{16 \rho R}\left(1-8 \beta_{1} R^{2}\right)+\cdots\right] \\
& =\frac{L_{\text {AdS }}^{3}(1-6 \alpha \lambda)}{4 G_{N}^{(5)}} \frac{2 \pi l R}{2 \epsilon^{2}}+\frac{L_{\text {AdS }}^{3}}{4 G_{N}^{(5)}} \frac{2 \pi l}{R} \frac{1-2 \alpha \lambda}{8}\left(1-8 \beta_{1} R^{2}\right) \ln \epsilon+\cdots
\end{aligned}
$$

Notice that the coefficient of the logarithmic term differs by a factor equal to $\left(1-8 \beta_{1} R^{2}\right)$ compared to the conformal case. Depending on the sign of $\beta_{1}$, the overall coefficient behaves in exactly the same way for both the ball and the cylinder. It will be interesting to understand the implications of this statement.

\section{3 $\beta_{1}$ and the weak energy condition}

Here we will examine the weak (or null) energy condition and investigate whether it is possible to constrain the sign of $\beta_{1}$ without further specifying the geometry (7.2). The nullenergy condition implies that the matter stress energy tensor $T_{M N}$ satisfies $T_{M N} \zeta^{M} \zeta^{N} \geq 0$ for any arbitrary null vector $\zeta^{M}$. Consider the most general null vector $\zeta^{M}$

$$
g_{\mu \nu} \zeta^{\mu} \zeta^{\nu}=0 \Rightarrow\left(\zeta^{\rho}\right)^{2}=-4 \rho e^{2 U(\rho)}\left(\eta_{i j} \zeta^{i} \zeta^{j}\right)
$$

where $\eta_{i j}$ represents the four dimensional Minkowski metric. It directly follows from (7.11) that $\left(\eta_{i j} \zeta^{i} \zeta^{j}\right) \leq 0$. Substituting eq. (7.11) into the null energy condition yields

$$
T_{\mu \nu} \zeta^{\mu} \zeta^{\nu} \geq 0 \Rightarrow-\left(\eta_{i j} \zeta^{i} \zeta^{j}\right)\left(T_{t t}+4 \rho e^{2 U(\rho)} T_{\rho \rho}\right) \geq 0
$$

Here ${ }^{15}$ we used $T_{x^{i} x^{i}}=-T_{t t}$ which follows from the symmetries of the metric (7.2). As we saw earlier $-\eta_{i j} \zeta^{i} \zeta^{j} \geq 0$ which results in $\left(T_{t t}+4 \rho e^{2 U(\rho)} T_{\rho \rho}\right) \geq 0$.

Recall that the equations of motion (3.4) for $d=4$ in the presence of matter reduce to

$$
-\frac{1}{2} g_{M N} \mathcal{L}+R_{M N}+\lambda L^{2} \mathcal{H}_{M N}^{(2)}=\left(8 \pi G_{N}^{(5)}\right) T_{M N}
$$

Eq. (7.13) allows the determination of $\left(T_{t t}+4 \rho e^{2 U(\rho)} T_{\rho \rho}\right)$ for arbitrary matter sector

$$
\begin{aligned}
T_{t t}+4 \rho e^{2 U(\rho)} T_{\rho \rho}= & -12 e^{2 U(\rho)}\left(U^{\prime}(\rho)+\rho U^{\prime \prime}(\rho)\right) \times \\
& \times\left(1-2 \lambda \alpha+8 \lambda \alpha \rho U^{\prime}(\rho)\left[1-\rho U^{\prime}(\rho)\right]\right) .
\end{aligned}
$$

\footnotetext{
${ }^{15}$ Note that $(7.12)$ can also be written as $-\frac{e^{2 U(\rho)}}{\rho}\left(\eta_{i j} \zeta^{i} \zeta^{j}\right)\left(-T_{t}^{t}+T_{\rho}^{\rho}\right)$.
} 
The energy condition implies that the left hand side is positive for any $\rho$, therefore for $\rho=0$ as well. The Fefferman-Graham expansion (7.3) helps us evaluate (7.14) at $\rho=0$ and combined with the null-energy condition leads to

$$
6 \beta_{1} \frac{\alpha-2}{\alpha} \geq 0
$$

with $\alpha=\left(\frac{L}{L}\right)^{2}$. To leading order close to the boundary $\alpha$ is defined by (3.7). For the stable AdS solution of Gauss-Bonnet gravity $(\alpha-2)>0$ fixes the sign of $\beta_{1}$ to be negative. This is exactly what happens in Einstein-Hilbert gravity with a cosmological constant. It is interesting that had we chosen the unstable AdS solution we would have a positive $\beta_{1}$.

We conclude that the coefficient of the logarithmically divergent term in the entropy of both the ball and the infinite cylinder is monotonically increasing with dilatations (as long as $\beta_{1}$ is non-vanishing). Unfortunately, this does not mean much. To apply the reasoning of $[94,95]$ the full result, not just the logarithmic term, is required; mainly because away from the conformal fixed point the EE depends on two, rather than one length scale, i.e., the radius $R$ of the ball and the scale of the theory $\mu$.

\subsection{Entanglement entropy of the ball and the c-theorem in Einstein-Hilbert gravity}

In the previous section we saw that the coefficient of the logarithmically divergent term in the entanglement entropy depends only on the UV data of the theory. It is therefore clear that this coefficient is not a good candidate for a function decreasing along the RG flow and being equal to $a$ at the fixed points. This result, together with the work of Casini and Huerta [42] in two-dimensional field theories, lead us to consider instead the following quantity

$$
Q \equiv-R \frac{\partial S_{E E, r e g}}{\partial R} \rightarrow a_{C F T}
$$

Here $S_{E E \text {,reg }}$ contains only the finite part of the entanglement entropy of a ball of radius $R$. We would like to examine the monotonicity properties of $Q(R)$ holographically. A priori, this does not seem an easy task since we cannot compute (7.16) exactly in an arbitrary domain wall geometry. However, it is possible that the null energy condition and general characteristics of the spacetime (7.2) will determine whether (7.16) behaves monotonically along renormalization group trajectories.

In the following we will attack this problem in the context of Einstein-Hilbert gravity. This has the advantage of being technically simpler while the relevant features of the problem remain the same. To simplify the analysis we will parametrize the surface in the bulk by $\rho\left(x^{i}\right)$ where $x^{i}$ for $i=1,2,3$ are cartesian coordinates at the boundary. This choice yields a lagrangian independent of $x^{i}$

$$
\begin{aligned}
S(B) & =\frac{\widetilde{L}^{3}}{4 G_{N}^{(5)}} \int \prod_{i=1}^{3} d x_{i} \mathcal{L}\left(\rho\left(x^{i}\right), \partial_{i} \rho\left(x^{i}\right)\right) \\
\mathcal{L} & =\frac{e^{2 U(\rho)}}{2 \rho\left(x^{i}\right)^{2}} \sqrt{\sum_{i}\left[\partial_{i} \rho\left(x^{i}\right)\right]^{2}+4 e^{2 U(\rho)} \rho\left(x^{i}\right)}
\end{aligned}
$$


where $\rho\left(x^{i}\right)$ determines the profile of the embedding surface. Translational invariance implies that the system has a conserved "stress-energy" tensor $T_{i}^{j}$

$$
T_{i}^{j}=\frac{\partial \mathcal{L}}{\partial\left(\partial_{j} \rho\right)}\left(\partial_{i} \rho\right)-\mathcal{L} \delta_{i}^{j}
$$

obeying $\partial_{i} T_{i}^{j}=0$. Due to spherical symmetry $T_{i j}$ takes the form

$$
T^{i j}=A(r) \widehat{r}^{i} \widehat{r}^{j}+\delta^{i j} B(r)
$$

where $A, B$ can be explicitly found to be

$$
\begin{aligned}
& A(r)=\frac{e^{2 U(\rho)}}{2 \rho^{2}} \frac{\rho^{\prime}(r)^{2}}{\sqrt{\rho^{\prime}(r)^{2}+4 e^{2 U(\rho)} \rho}}=\frac{e^{2 U(\rho)}}{2 \rho^{2}} \frac{1}{\left|r^{\prime}(\rho)\right| \sqrt{1+4 e^{2 U(\rho)} \rho r^{\prime}(\rho)^{2}}} \\
& B(r)=-\frac{e^{2 U(\rho)}}{2 \rho^{2}} \sqrt{\rho^{\prime}(r)^{2}+4 e^{2 U(\rho)} \rho r^{\prime}(\rho)^{2}}=-\frac{e^{2 U(\rho)}}{2 \rho^{2}\left|r^{\prime}(\rho)\right|} \sqrt{1+4 e^{2 U(\rho)} \rho r^{\prime}(\rho)^{2}}
\end{aligned}
$$

In the first equality we have replaced the dependence of $\rho$ on the cartesian coordinates $x^{i}$ with the spherical coordinate $r$. In the second equality we give $A(r), B(r)$ in terms of $r(\rho)$ instead of $\rho(r)$. Expressing the geometry of the embedding surface with $r(\rho)$ will be more convenient in the following. Note that $r(\rho)$ satisfies the conservation equation of $T^{i j}$, in other words the equations of motion derived from (7.17). The conservation equation can be written in a simple form in terms of the functions $A(r)$ and $B(r)$

$$
2 A(r)+r \frac{d}{d r}(A(r)+B(r))=0
$$

Solving for $r(\rho)$ in the vicinity of the UV with the help of (7.3) and (7.20) yields

$$
r(\rho)=R-\frac{\rho}{2 R}+\sigma_{1} \rho^{2}+\frac{\beta_{1}}{4 R} \rho^{2} \log \rho+\sigma_{3} \rho^{3} \cdots .
$$

where $\sigma_{1}$ is a function of the parameters $\left(\beta_{1}, \beta_{2}, \beta_{3}\right)$ which specify the domain wall geometry but also of $\sigma_{3}$, the coefficient of a higher order term in the near boundary expansion of $r(\rho)$

$$
\sigma_{1}=\frac{9-27 R^{2} \beta_{1}+4 R^{4}\left(2 \beta_{1}^{2}+9 \beta_{2}-6 \beta_{3}\right)-72 R^{5} \sigma_{3}}{12 R^{3}\left(-9+8 R^{2} \beta_{1}\right)} .
$$

Unfortunately, $\sigma_{1}$ cannot be determined without knowledge of the exact form of the profile function $r(\rho)$. This will turn out to be the main difficulty in determining the behavior of $Q(R)$ under rescalings of $R$.

Now suppose we vary the boundary conditions in the entanglement entropy computation as follows

$$
\rho\left(x^{i}+\delta x^{i}\right)=\epsilon+\delta \epsilon
$$

where the original boundary condition is $\rho\left(x^{i}\right)=\epsilon$ for $x^{i} \in \mathcal{D}$ (here $\mathcal{D}$ is a sphere of radius $R$ ). The change in the on-shell action would then be equal to

$$
\delta S_{\text {on-shell }}=\frac{\widetilde{L}^{3}}{4 G_{N}^{(5)}}\left[\int_{\mathcal{D}} d \Sigma_{i} \frac{\delta \mathcal{L}}{\delta \partial_{i} \rho} \delta \epsilon-\int_{\mathcal{D}} d \Sigma_{j} T_{i}^{j} \delta x^{i}\right]
$$


with $d \Sigma_{i}$ the volume element on $\mathcal{D}$. Substituting (7.19) into (7.25) leads to

$$
Q=-R \frac{\partial S_{E E, \text { reg }}}{\partial R}==\left.\frac{\widetilde{L}^{3} \Omega_{2}}{4 G_{N}^{(5)}} R^{3}(A(R)+B(R))\right|_{\text {reg }}
$$

Having obtained the explicit form of the divergences in the previous section, it is easy to subtract them from $A(R)+B(R)$ to arrive at

$$
Q=-R \frac{\partial S_{E E, r e g}}{\partial R}=\frac{\widetilde{L}^{3} \Omega_{2}}{4 G_{N}^{(5)}} \frac{1}{2}\left(1-3 R^{2} \beta_{1}+8 R^{3} \sigma_{1}\right)
$$

Although we have obtained a simple expression for $Q(R)$, we cannot determine whether it has a monotonic behavior under rescalings of the radius $R$. It appears that one would need to know the exact solution for the profile $r(\rho)$. Smoothness and other generic characteristics of the geometry of the induced surface did not suffice to prove the monotonicity of $Q(R)$.

Moreover, it is not clear how the null energy condition, pertinent to the ambient spacetime, will affect the behavior of $Q(r)$ which depends on the details of the embedding function $r(\rho)$. It is likely that even if $Q(R)$ is a c-function in the sense of Zamolodchikov, it is most likely a different c-function than the standard holographic one - which in the coordinates used here is expressed as $c_{\text {hol }} \sim \frac{1}{\left(-2 U^{\prime}(\rho) \rho+1\right)^{3}}$. On the other hand, the null energy condition may not be a sufficiently strong condition to establish a holographic ctheorem in the generic case. As illustrated in [10] for a simple scalar coupling, the null energy condition does not, at least in an obvious way, lead to a c-theorem.

It is also possible that our basic assumption, which is that the entanglement entropy computation is not altered by the presence of matter fields, is incorrect even when matter is coupled to gravity in the simplest, standard way (standard kinetic terms, no conformal couplings etc.).

Finally, we would like to note that the proof of [94] -[95] in two dimensions, is based on two main physical properties: Lorentz invariance of the theory and strong subadditivity of entanglement entropy. To make use of the latter, it might be more appropriate to consider the geometry of an annulus. We leave this issue to future investigation.

\section{Acknowledgments}

We would like to thank R. Myers, I. Papadimitriou, M. Rangamani and M. Taylor for useful discussions and H. Casini, M. Huerta and particularly S. Solodukhin for helpful correspondence. We are grateful to the organizers of the "Crete Conference on Gauge Theories and Structure of Spacetime" for a great working atmosphere. M.K. thanks Galileo Galilei Institute in Florence, and University of Barcelona and A.P. thanks Galileo Galilei Institute in Florence, University of Chicago and Uppsala University for hospitality during the completion of this work. The work of M.K. is supported by the Göran Gustaffson Foundation.

Open Access. This article is distributed under the terms of the Creative Commons Attribution Noncommercial License which permits any noncommercial use, distribution, and reproduction in any medium, provided the original author(s) and source are credited. 


\section{References}

[1] S. Ryu and T. Takayanagi, Holographic derivation of entanglement entropy from AdS/CFT, Phys. Rev. Lett. 96 (2006) 181602 [hep-th/0603001] [SPIRES].

[2] S. Ryu and T. Takayanagi, Aspects of holographic entanglement entropy, JHEP 08 (2006) 045 [hep-th/0605073] [SPIRES].

[3] D.M. Hofman and J. Maldacena, Conformal collider physics: energy and charge correlations, JHEP 05 (2008) 012 [arXiv:0803.1467] [SPIRES].

[4] A.B. Zamolodchikov, Irreversibility of the flux of the renormalization group in a $2 D$ field theory, JETP Lett. 43 (1986) 730 [Pisma Zh. Eksp. Teor. Fiz. 43 (1986) 565] [SPIRES].

[5] J.L. Cardy, Is there a c theorem in four-dimensions?, Phys. Lett. B 215 (1988) 749 [SPIRES].

[6] D. Erkal and D. Kutasov, a-maximization, global symmetries and $R G$ flows, arXiv: 1007.2176 [SPIRES].

[7] D. Poland and D. Simmons-Duffin, Bounds on $4 D$ conformal and superconformal field theories, JHEP 05 (2011) 017 [arXiv: 1009.2087] [SPIRES].

[8] D. Gaiotto, N. Seiberg and Y. Tachikawa, Comments on scaling limits of $4 D N=2$ theories, JHEP 01 (2011) 078 [arXiv:1011.4568] [SPIRES].

[9] R.C. Myers and A. Sinha, Seeing a c-theorem with holography, Phys. Rev. D 82 (2010) 046006 [arXiv: 1006.1263] [SPIRES].

[10] R.C. Myers and A. Sinha, Holographic c-theorems in arbitrary dimensions, JHEP 01 (2011) 125 [arXiv:1011.5819] [SPIRES].

[11] J.T. Liu, W. Sabra and Z. Zhao, Holographic c-theorems and higher derivative gravity, arXiv: 1012.3382 [SPIRES].

[12] M. Brigante, H. Liu, R.C. Myers, S. Shenker and S. Yaida, Viscosity bound violation in higher derivative gravity, Phys. Rev. D 77 (2008) 126006 [arXiv:0712.0805] [SPIRES].

[13] M. Brigante, H. Liu, R.C. Myers, S. Shenker and S. Yaida, The viscosity bound and causality violation, Phys. Rev. Lett. 100 (2008) 191601 [arXiv:0802.3318] [SPIRES].

[14] A. Buchel and R.C. Myers, Causality of holographic hydrodynamics, JHEP 08 (2009) 016 [arXiv:0906.2922] [SPIRES].

[15] D.M. Hofman, Higher derivative gravity, causality and positivity of energy in a UV complete QFT, Nucl. Phys. B 823 (2009) 174 [arXiv:0907.1625] [SPIRES].

[16] J. de Boer, M. Kulaxizi and A. Parnachev, AdS $S_{7} / C F T_{6}$, Gauss-Bonnet gravity and viscosity bound, JHEP 03 (2010) 087 [arXiv:0910.5347] [SPIRES].

[17] X.O. Camanho and J.D. Edelstein, Causality constraints in AdS/CFT from conformal collider physics and Gauss-Bonnet gravity, JHEP 04 (2010) 007 [arXiv:0911.3160] [SPIRES].

[18] A. Buchel et al., Holographic GB gravity in arbitrary dimensions, JHEP 03 (2010) 111 [arXiv:0911.4257] [SPIRES].

[19] J. de Boer, M. Kulaxizi and A. Parnachev, Holographic Lovelock gravities and black holes, JHEP 06 (2010) 008 [arXiv:0912.1877] [SPIRES].

[20] X.O. Camanho and J.D. Edelstein, Causality in AdS/CFT and Lovelock theory, JHEP 06 (2010) 099 [arXiv:0912.1944] [SPIRES]. 
[21] R.C. Myers, M.F. Paulos and A. Sinha, Holographic studies of quasi-topological gravity, JHEP 08 (2010) 035 [arXiv: 1004.2055] [SPIRES].

[22] M. Kulaxizi and A. Parnachev, Energy flux positivity and unitarity in CFTs, Phys. Rev. Lett. 106 (2011) 011601 [arXiv:1007.0553] [SPIRES].

[23] A. Buchel and S. Cremonini, Viscosity bound and causality in superfluid plasma, JHEP 10 (2010) 026 [arXiv:1007.2963] [SPIRES].

[24] R.C. Myers, S. Sachdev and A. Singh, Holographic quantum critical transport without self-duality, Phys. Rev. D 83 (2011) 066017 [arXiv: 1010.0443] [SPIRES].

[25] X.O. Camanho, J.D. Edelstein and M.F. Paulos, Lovelock theories, holography and the fate of the viscosity bound, JHEP 05 (2011) 127 [arXiv:1010.1682] [SPIRES].

[26] S.N. Solodukhin, Entanglement entropy, conformal invariance and extrinsic geometry, Phys. Lett. B 665 (2008) 305 [arXiv:0802.3117] [SPIRES].

[27] D.V. Fursaev, Proof of the holographic formula for entanglement entropy, JHEP 09 (2006) 018 [hep-th/0606184] [SPIRES].

[28] L.-Y. Hung, R.C. Myers and M. Smolkin, On holographic entanglement entropy and higher curvature gravity, JHEP 04 (2011) 025 [arXiv: 1101.5813] [SPIRES].

[29] H. Casini, M. Huerta and R.C. Myers, Towards a derivation of holographic entanglement entropy, JHEP 05 (2011) 036 [arXiv:1102.0440] [SPIRES].

[30] L. Bombelli, R.K. Koul, J. Lee and R.D. Sorkin, A quantum source of entropy for black holes, Phys. Rev. D 34 (1986) 373 [SPIRES].

[31] M. Srednicki, Entropy and area, Phys. Rev. Lett. 71 (1993) 666 [hep-th/9303048] [SPIRES].

[32] M.B. Plenio, J. Eisert, J. Dreissig and M. Cramer, Entropy, entanglement and area: analytical results for harmonic lattice systems, Phys. Rev. Lett. 94 (2005) 060503 [quant-ph/0405142] [SPIRES].

[33] M. Cramer, J. Eisert, M.B. Plenio and J. Dreissig, An entanglement-area law for general bosonic harmonic lattice systems, Phys. Rev. A 73 (2006) 012309 [quant-ph/0505092] [SPIRES].

[34] M. Ahmadi, S. Das and S. Shankaranarayanan, Is entanglement entropy proportional to area?, Can. J. Phys. 84 (2006) 493 [hep-th/0507228] [SPIRES].

[35] S. Das and S. Shankaranarayanan, How robust is the entanglement entropy-area relation?, Phys. Rev. D 73 (2006) 121701 [gr-qc/0511066] [SPIRES].

[36] H. Casini, Geometric entropy, area and strong subadditivity, Class. Quant. Grav. 21 (2004) 2351 [hep-th/0312238] [SPIRES].

[37] H. Casini and M. Huerta, Analytic results on the geometric entropy for free fields, J. Stat. Mech. (2008) P01012 [arXiv:0707.1300] [SPIRES].

[38] M.M. Wolf, Violation of the entropic area law for fermions, Phys. Rev. Lett. 96 (2004) 010404 [quant-ph/0503219].

[39] D. Gioev and I. Klich, Entanglement entropy of fermions in any dimension and the Widom conjecture, Phys. Rev. Lett. 96 (2006) 100503 [quant-ph/0504151].

[40] T. Barthel, M. Chung and U. Schollwoeck, Entanglement scaling in critical two-dimensional fermionic and bosonic systems, Phys. Rev. A 74 (2006) 022329 [cond-mat/0602077].

[41] W. Li, L. Leitian Ding, R. Yu, T. Roscilde and S. Haas, Scaling behavior of entanglement in two- and three-dimensional free fermions, Phys. Rev. B 74 (2007) 0703103 [quant-ph/0602094]. 
[42] H. Casini and M. Huerta, Universal terms for the entanglement entropy in $2+1$ dimensions, Nucl. Phys. B 764 (2007) 183 [hep-th/0606256] [SPIRES].

[43] R. Lohmayer, H. Neuberger, A. Schwimmer and S. Theisen, Numerical determination of entanglement entropy for a sphere, Phys. Lett. B 685 (2010) 222 [arXiv:0911.4283] [SPIRES].

[44] H. Casini and M. Huerta, Entanglement entropy for the n-sphere, Phys. Lett. B 694 (2010) 167 [arXiv:1007.1813] [SPIRES].

[45] J.S. Dowker, Hyperspherical entanglement entropy, J. Phys. A 43 (2010) 445402 [arXiv: 1007.3865] [SPIRES].

[46] J.S. Dowker, Entanglement entropy for even spheres, arXiv:1009.3854 [SPIRES].

[47] J.S. Dowker, Determinants and conformal anomalies of GJMS operators on spheres, J. Phys. A 44 (2011) 115402 [arXiv:1010.0566] [SPIRES].

[48] J.S. Dowker, Entanglement entropy for odd spheres, arXiv: 1012.1548 [SPIRES].

[49] Q. Exirifard and M.M. Sheikh-Jabbari, Lovelock gravity at the crossroads of Palatini and metric formulations, Phys. Lett. B 661 (2008) 158 [arXiv:0705.1879] [SPIRES].

[50] D.G. Boulware and S. Deser, String generated gravity models, Phys. Rev. Lett. 55 (1985) 2656 [SPIRES].

[51] R.-G. Cai, Gauss-Bonnet black holes in AdS spaces, Phys. Rev. D 65 (2002) 084014 [hep-th/0109133] [SPIRES].

[52] M. Henningson and K. Skenderis, The holographic Weyl anomaly, JHEP 07 (1998) 023 [hep-th/9806087] [SPIRES].

[53] M. Henningson and K. Skenderis, Holography and the Weyl anomaly, Fortsch. Phys. 48 (2000) 125 [hep-th/9812032] [SPIRES].

[54] S. Nojiri and S.D. Odintsov, On the conformal anomaly from higher derivative gravity in AdS/CFT correspondence, Int. J. Mod. Phys. A 15 (2000) 413 [hep-th/9903033] [SPIRES].

[55] V.E. Hubeny, M. Rangamani and T. Takayanagi, A covariant holographic entanglement entropy proposal, JHEP 07 (2007) 062 [arXiv:0705.0016] [SPIRES].

[56] T. Nishioka, S. Ryu and T. Takayanagi, Holographic entanglement entropy: an overview, J. Phys. A 42 (2009) 504008 [arXiv: 0905.0932] [SPIRES].

[57] M. Headrick and T. Takayanagi, A holographic proof of the strong subadditivity of entanglement entropy, Phys. Rev. D 76 (2007) 106013 [arXiv:0704.3719] [SPIRES].

[58] C. Holzhey, F. Larsen and F. Wilczek, Geometric and renormalized entropy in conformal field theory, Nucl. Phys. B 424 (1994) 443 [hep-th/9403108] [SPIRES].

[59] P. Calabrese and J.L. Cardy, Entanglement entropy and quantum field theory, J. Stat. Mech. (2004) P06002 [hep-th/0405152] [SPIRES].

[60] P. Calabrese and J.L. Cardy, Entanglement entropy and quantum field theory: a non-technical introduction, Int. J. Quant. Inf. 4 (2006) 429 [quant-ph/0505193] [SPIRES].

[61] J.-R. Sun, Note on Chern-Simons term correction to holographic entanglement entropy, JHEP 05 (2009) 061 [arXiv:0810.0967] [SPIRES].

[62] D.V. Fursaev and S.N. Solodukhin, On the description of the Riemannian geometry in the presence of conical defects, Phys. Rev. D 52 (1995) 2133 [hep-th/9501127] [SPIRES].

[63] D.V. Fursaev and S.N. Solodukhin, On one loop renormalization of black hole entropy, Phys. Lett. B 365 (1996) 51 [hep-th/9412020] [SPIRES]. 
[64] S.N. Solodukhin, On 'non-geometric' contribution to the entropy of black hole due to quantum corrections, Phys. Rev. D 51 (1995) 618 [hep-th/9408068] [SPIRES].

[65] S.N. Solodukhin, The conical singularity and quantum corrections to entropy of black hole, Phys. Rev. D 51 (1995) 609 [hep-th/9407001] [SPIRES].

[66] D.V. Fursaev, Black hole thermodynamics and renormalization, Mod. Phys. Lett. A 10 (1995) 649 [hep-th/9408066] [SPIRES].

[67] D.V. Fursaev, Spectral geometry and one loop divergences on manifolds with conical singularities, Phys. Lett. B 334 (1994) 53 [hep-th/9405143] [SPIRES].

[68] D. Nesterov and S.N. Solodukhin, Short-distance regularity of Green's function and UV divergences in entanglement entropy, JHEP 09 (2010) 041 [arXiv: 1008. 0777] [SPIRES].

[69] T. Padmanabhan, Finite entanglement entropy from the zero-point-area of spacetime, Phys. Rev. D 82 (2010) 124025 [arXiv:1007.5066] [SPIRES].

[70] D. Nesterov and S.N. Solodukhin, Gravitational effective action and entanglement entropy in UV modified theories with and without Lorentz symmetry, Nucl. Phys. B 842 (2011) 141 [arXiv: 1007.1246] [SPIRES].

[71] M. Headrick, Entanglement Renyi entropies in holographic theories, Phys. Rev. D 82 (2010) 126010 [arXiv: 1006.0047] [SPIRES].

[72] T. Jacobson and R.C. Myers, Black hole entropy and higher curvature interactions, Phys. Rev. Lett. 70 (1993) 3684 [hep-th/9305016] [SPIRES].

[73] M. Bañados, C. Teitelboim and J. Zanelli, Black hole entropy and the dimensional continuation of the Gauss-Bonnet theorem, Phys. Rev. Lett. 72 (1994) 957 [gr-qc/9309026] [SPIRES].

[74] W. Nelson, A comment on black hole entropy in string theory, Phys. Rev. D 50 (1994) 7400 [hep-th/9406011] [SPIRES].

[75] V. Iyer and R.M. Wald, A comparison of Noether charge and euclidean methods for computing the entropy of stationary black holes, Phys. Rev. D 52 (1995) 4430 [gr-qc/9503052] [SPIRES].

[76] I.R. Klebanov, D. Kutasov and A. Murugan, Entanglement as a probe of confinement, Nucl. Phys. B 796 (2008) 274 [arXiv:0709.2140] [SPIRES].

[77] M. Kulaxizi and A. Parnachev, Supersymmetry constraints in holographic gravities, Phys. Rev. D 82 (2010) 066001 [arXiv:0912.4244] [SPIRES].

[78] S. Kachru, X. Liu and M. Mulligan, Gravity duals of Lifshitz-like fixed points, Phys. Rev. D 78 (2008) 106005 [arXiv:0808.1725] [SPIRES].

[79] K. Balasubramanian and K. Narayan, Lifshitz spacetimes from AdS null and cosmological solutions, JHEP 08 (2010) 014 [arXiv: 1005.3291] [SPIRES].

[80] A. Donos and J.P. Gauntlett, Lifshitz solutions of $D=10$ and $D=11$ supergravity, JHEP 12 (2010) 002 [arXiv: 1008.2062] [SPIRES].

[81] A. Donos, J.P. Gauntlett, N. Kim and O. Varela, Wrapped M5-branes, consistent truncations and AdS/CMT, JHEP 12 (2010) 003 [arXiv: 1009.3805] [SPIRES].

[82] R. Gregory, S.L. Parameswaran, G. Tasinato and I. Zavala, Lifshitz solutions in supergravity and string theory, JHEP 12 (2010) 047 [arXiv: 1009.3445] [SPIRES].

[83] A. Adams, A. Maloney, A. Sinha and S.E. Vazquez, $1 / N$ effects in non-relativistic gauge-gravity duality, JHEP 03 (2009) 097 [arXiv: 0812.0166] [SPIRES]. 
[84] M.H. Dehghani and R.B. Mann, Lovelock-Lifshitz black holes, JHEP 07 (2010) 019 [arXiv: 1004.4397] [SPIRES].

[85] M.H. Dehghani and R.B. Mann, Thermodynamics of Lovelock-Lifshitz black branes, Phys. Rev. D 82 (2010) 064019 [arXiv:1006.3510] [SPIRES].

[86] A.H. Chamseddine, Topological gauge theory of gravity in five-dimensions and all odd dimensions, Phys. Lett. B 233 (1989) 291 [SPIRES].

[87] S.N. Solodukhin, Entanglement entropy in non-relativistic field theories, JHEP 04 (2010) 101 [arXiv:0909.0277] [SPIRES].

[88] T. Azeyanagi, W. Li and T. Takayanagi, On string theory duals of Lifshitz-like fixed points, JHEP 06 (2009) 084 [arXiv:0905.0688] [SPIRES].

[89] C. Hoyos and P. Koroteev, On the null energy condition and causality in Lifshitz holography, Phys. Rev. D 82 (2010) 084002 [Erratum ibid. D 82 (2010) 109905] [arXiv:1007.1428] [SPIRES].

[90] M. Berg and H. Samtleben, An exact holographic RG flow between $2 D$ conformal fixed points, JHEP 05 (2002) 006 [hep-th/0112154] [SPIRES].

[91] M. Taylor, More on counterterms in the gravitational action and anomalies, hep-th/0002125 [SPIRES].

[92] M. Bianchi, D.Z. Freedman and K. Skenderis, Holographic renormalization, Nucl. Phys. B 631 (2002) 159 [hep-th/0112119] [SPIRES].

[93] I. Papadimitriou and K. Skenderis, AdS/CFT correspondence and geometry, hep-th/0404176 [SPIRES].

[94] H. Casini and M. Huerta, A finite entanglement entropy and the c-theorem, Phys. Lett. B 600 (2004) 142 [hep-th/0405111] [SPIRES].

[95] H. Casini and M. Huerta, A c-theorem for the entanglement entropy, J. Phys. A 40 (2007) 7031 [cond-mat/0610375] [SPIRES]. 